\title{
Enfermedad de Paget extramamaria perianal
}

\author{
Nathalia Córdoba Ramírez'; María Pineda Muñoz²; Andrés Felipe Toro Palacio; \\ Julia Mesa Villegas ${ }^{4}$
}

\section{RESUMEN}

La enfermedad de Paget constituye un adenocarcinoma intraepidérmico, que afecta principalmente al pezón y, más raramente, a otras zonas apocrinas extramamarias. La localización perianal representa el $20 \%$ de los casos extramamarios y el 1\% de las neoplasias perianales. En general, es más frecuente en mujeres posmenopáusicas.

Se presenta el caso clínico de una paciente joven, que desarrolla una placa eritematodescamativa persistente en la región perianal. Se toma biopsia sin sospechar la condición; mediante histopatología e inmunohistoquímica se confirma el diagnóstico de enfermedad de Paget extramamaria. Se descarta compromiso en otros órganos y se realiza tratamiento quirúrgico, el cual es exitoso hasta la fecha.

PALABRAS CLAVE: enfermedad de Paget extramamaria; neoplasias de las glándulas anales; adenocarcinoma; glándulas Perianales.

\section{EXTRAMAMMARY PAGET'S DISEASE OF PERIANAL REGIÓN}

\section{SUMMARY}

Paget's disease constitutes an intraepidermal adenocarcinoma that affects the nipple and more rarely, other extra-mammary apocrine areas. The perianal location represents $20 \%$ of extramammary cases and $1 \%$ of perianal neoplasms. In general, it is more common in postmenopausal women.

We present the clinical case of a young patient that develops a persistent plaque in the perianal region. A biopsy is taken, without suspecting the entity, and through histopathology and immunohistochemistry the diagnosis of Paget's extramammary disease is confirmed. Compromise in other organs is ruled out and a successful surgical treatment is carried out.

KEY WORDS: Paget disease; Extramammary; Anal gland neoplasms; Adenocarcinoma; Perianal glands.

1. Médica, residente de tercer año de Dermatología. Universidad de Caldas, Manizales. ORCID https://orcid.org/oooo-0oo1-7540-3451

2. Estudiante de Medicina, Universidad de Caldas, Manizales.ORCID https://orcid.org/oooo-0001-5305-4182

3. Estudiante de Medicina, Universidad de Caldas, Manizales.ORCID https://orcid.org/oooo-0003-2156-5046

4. Médica dermatóloga; dermatopatóloga; docente. Universidad de Caldas, Manizales. ORCID https://orcid.org/oooo-0002-7413-121X

Correspondencia: Nathalia Córdoba Ramírez; email: Inatika1@hotmail.com

Recibido:08/03/20; aceptado: 26/04/20

Cómo citar: Córdoba-Ramírez N, Pineda-Muñoz M, Toro-Palacio AF, Mesa-Villegas J. Enfermedad de Paget extramamaria perianal. Rev Asoc Colomb Dermatol. 2020;28(1):75-81. DOI: https://doi.org/10.29176/2590843X.1492

Financiación: ninguna, conflictos de interés: ninguno 


\section{INTRODUCCIÓN}

La enfermedad de Paget es un adenocarcinoma intraepidérmico clasificado como mamario o extramamario, el cual afecta principalmente al pezón y, con menor frecuencia, a otras zonas apocrinas. Ambas formas poseen similitudes clínicas e histológicas, pero se distinguen por la frecuencia con que se asocian a otros tipos de cáncer, su pronóstico y tratamiento ${ }^{(1,2)}$.

Clínicamente se presenta como una lesión eritematosa, eccematosa, costrosa y húmeda con o sin descamación fina. El diagnóstico es histológico y se confirma mediante inmunohistoquímica ${ }^{(3)}$.

La forma mamaria se relaciona en más del $90 \%$ de los casos con cáncer de mama subyacente y una de las grandes dificultades es que tiende a ser multifocal y multicéntrica, por lo que se debe realizar, además de mamografía, resonancia magnética para determinar la extensión real de la enfermedad (4).

La forma extramamaria tiene una asociación variable con otros tipos de cáncer; de manera global, es menor del 30\%, aunque la localizada a nivel perianal cuenta con tasas más altas. Los tumores de vulva, vagina, cérvix, cuerpo del útero, vejiga, ovarios, vesícula biliar, hígado, colon y recto son los más relacionados. Es mandatorio descartar una neoplasia subyacente, realizando estudios basados en la revisión por sistemas y el examen físico. Se ha propuesto realizar un tamizaje con citología urinaria, colonoscopia para ambos sexos y, en las mujeres, adicionar citología vaginal y mamografía; en los hombres, antígeno prostático ${ }^{(5)}$.

El tratamiento de elección es quirúrgico, mediante escisión amplia o cirugía micrográfica de Mohs ${ }^{(3)}$.

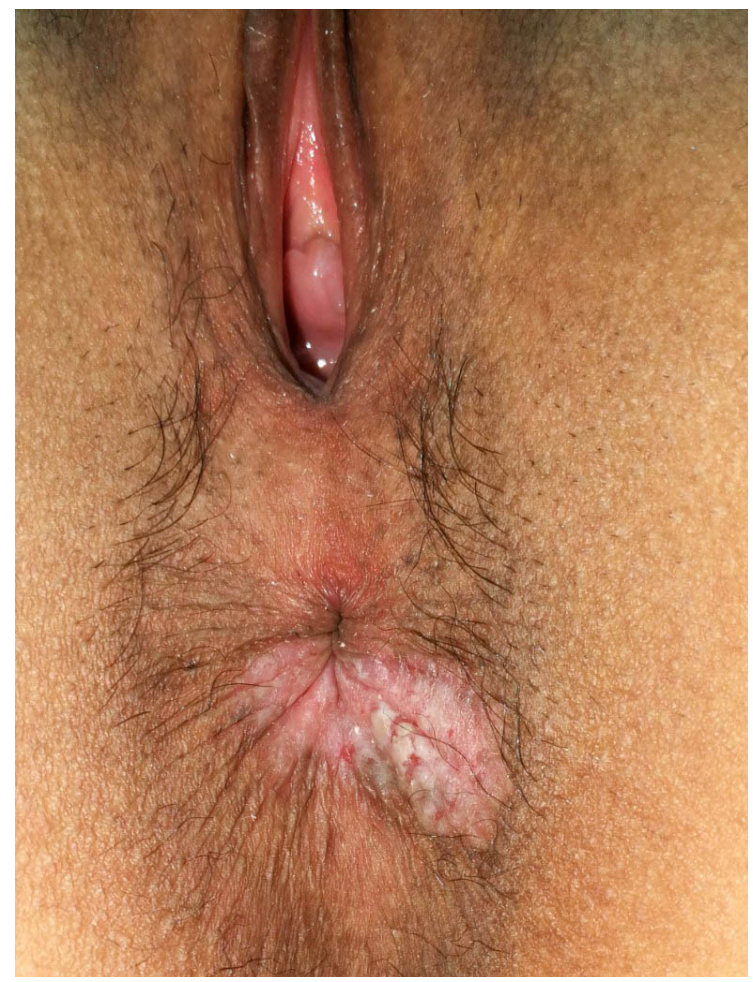

Figura 1. Placa de $4 \times 2 \mathrm{~cm}$ eritematoescamosa y erosionada en la piel perianal, con extensión a la unión mucocutánea, sin aparente infiltración dérmica. 


\section{PRESENTACIÓN DEL CASO}

Paciente femenina de 40 años, sin antecedentes patológicos de importancia, con cuadro clínico de aproximadamente 1 año de evolución, consistente con la aparición de lesión pruriginosa con erosión frecuente a nivel perianal, para lo cual recibió múltiples manejos con antimicóticos y esteroides tópicos, sin mejoría.

Durante consulta con dermatología por otra causa, se encontró placa eritematoescamosa y erosionada en la región perianal, de $4 \times 2 \mathrm{~cm}$, que se extendía a la unión mucocutánea (figura 1). Se tomó biopsia por sospecha de enfermedad de Bowen con diagnóstico diferencial de enfermedad por virus del papiloma humano (VPH). La combinación de hallazgos histológicos y de inmunohistoquímica permitieron realizar el diagnóstico de enfermedad de Paget extramamaria (figura 2).

En los estudios de inmunohistoquímica, la proliferación expresó positividad para citoqueratina 7 (CK7), antígeno carcinoembrionario (CAE) y antígeno epitelial de membrana (EMA); los marcadores p63, Melan A y citoqueratina 20 (CK20) fueron negativos (figura 3). Con esto resultados se confirmó enfermedad de Paget extramamaria perianal.

Los exámenes de extensión, perfil bioquímico, mamografía, colonoscopia, TAC toraco-abdomino-pélvico y citología vaginal fueron normales para el momento del diagnóstico.

En vista de la edad de la paciente, tamaño y ubicación de la lesión, se decide realizar cirugía micrográfica de Mohs modificada con procesamiento por parafina. En el primer tiempo quirúrgico, se hace resección con margen de $1 \mathrm{~cm}$, que resulta en compromiso de los distintos márgenes de resección; la paciente es llevada a un segundo tiempo quirúrgico diferido, se da $1 \mathrm{~cm}$ adicional de margen en toda la circunferencia a partir de la cicatriz, se obtienen márgenes libres de compromiso tumoral confirmados histológicamente y con inmunohistoquímica. Hasta la fecha, 8 meses después de la cirugía no hay signos de recurrencia, la paciente está asintomática y con preservación del tono del esfínter anal.

\section{DISCUSIÓN}

La enfermedad de Paget es un adenocarcinoma intraepitelial, que afecta zonas anatómicas con abundantes glándulas sudoríparas apocrinas ${ }^{(1,6)}$. Se clasifica en mamaria y extramamaria, con base en su ubicación anatómica, y en primaria y secundaria, en relación con la presencia o ausencia de neoplasia subyacente (2). Es una enfermedad poco frecuente, cuya incidencia varía entre 0,1 y 2,4 pacientes por cada 1.000.00o de personas/año ${ }^{(7)}$.

La enfermedad de Paget fue descrita por primera vez por el médico cirujano Sir James Paget, en $1874^{(1)}$. La histopatología fue descrita por Butlin dos años después ${ }^{(2)}$. En 1889, Crocker reportó lesiones con similitud histológica a la enfermedad de Paget en el escroto y el pene, describiendo así por primera vez la enfermedad de Paget extramamaria ${ }^{(8)}$. Los sitios de mayor afectación extramamaria son vulva, pene, escroto, periné y área perianal. Otros menos comunes son axila, ombligo, párpados, conducto auditivo externo y localizaciones ectópicas en otras partes de la cabeza, el tronco y las extremidades ${ }^{(3)}$.

La fisiopatología precisa no está por completo dilucidada y es diferente para la enfermedad mamaria y la extramamaria, tanto primaria como secundaria. En la enfermedad de Paget mamaria existen dos teorías: la epidermotrópica y la de transformación maligna in situ. La primera sustenta que los cambios típicos de la enfermedad de Paget surgen en las células ductales y que estos se extenderían a lo largo de la membrana basal hasta el pezón; está respaldada por la amplia asociación con carcinoma ductal de mama. La segunda sostiene que la enfermedad de Paget se origina en las células epidérmicas del pezón por la transformación maligna de los queratinocitos; esta teoría explicaría los casos no asociados con un proceso neoplásico en la mama afectada ${ }^{(9-11)}$.

En la enfermedad de Paget extramamaria primaria se ha discutido que puede surgir de células pluripotenciales en la epidermis y/o anexos (posiblemente glándulas sudoríparas o folículos pilosos) ${ }^{(2)}$ y también se ha postulado el origen en las células de Toker, que son células claras relacionadas con la glándula mamaria, que se encuentran en el pezón y la vulva ${ }^{(7)}$. En cuanto a la secundaria, se acepta que se debe a la extensión directa de un carcinoma originado en los órganos adyacentes ${ }^{(3)}$.

La enfermedad de Paget perianal representa el 20\% de la enfermedad de Paget extramamaria, el 6,5\% de todas las enfermedades de Paget y solo el $1 \%$ de las neoplasias perianales. Afecta principalmente a las 

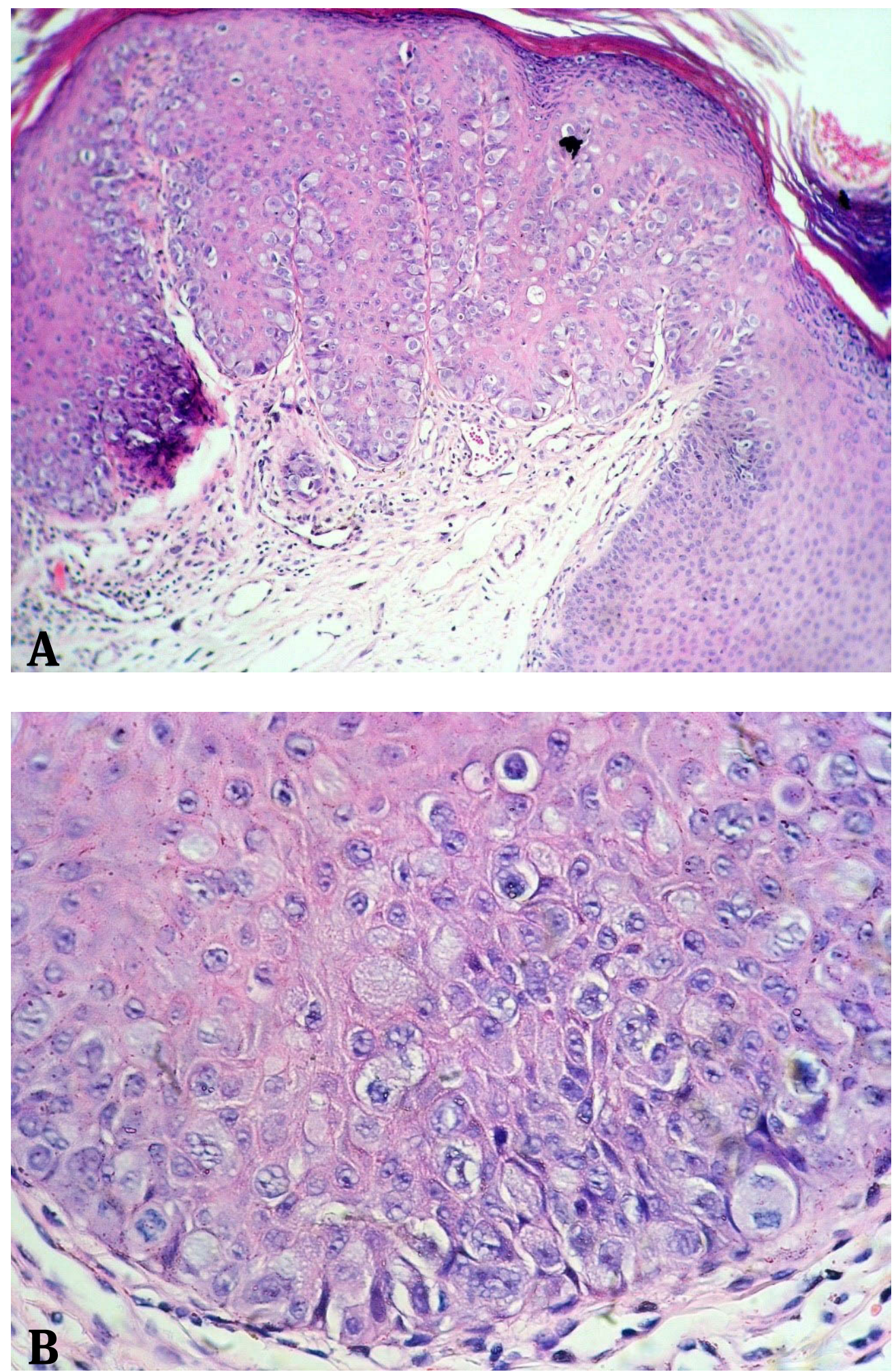

Figura 2. A) Biopsia de la lesión, en la que se observa hiperplasia epidérmica bulbosa, hipergranulosis e hiperqueratosis $10 \mathrm{X}$. B) Compromiso epidérmico por células claras de núcleos vesiculosos con heterocromasia y pleomorfismo, con citoplasmas amplios, pagetoides y claros. 40X. Hematoxilina-eosina. 

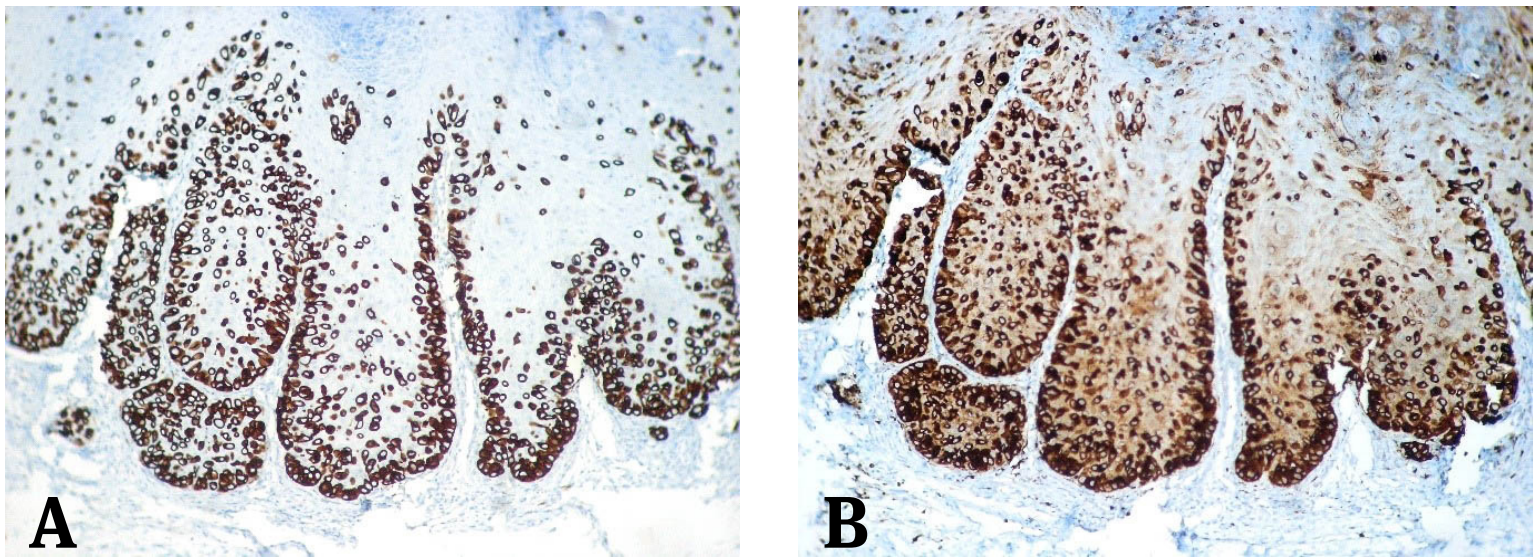

Figura 3. Inmunohistoquímica. A) Citoqueratina 7 positivo $\left(\mathrm{CK}_{7}\right)$. B) Antígeno carcinoembrionario positivo (CAE) $4 \mathrm{X}$.

mujeres posmenopáusicas entre los 50 a 80 años ${ }^{(12,13)}$. Darier y Coulillaud la describieron por primera vez en 1893. Usualmente se presenta como una placa eritematosa, eccematosa, descamativa, erosionada o ulcerada bien definida, asociada a prurito, dolor urente y, en algunas ocasiones, sangrado ${ }^{(12)}$. El diagnóstico de la enfermedad de Paget perianal se confirma por histología, inmunohistoquímica o microscopía electrónica. Son útiles múltiples marcadores, como el BerEp4, GCDFP15, CK20 y $\mathrm{CK}_{7}{ }^{\left({ }^{14}\right)}$; estos dos últimos utilizados para confirmar el diagnóstico en el caso que presentamos.

Como se mencionó, la enfermedad de Paget se relaciona con malignidad subyacente, que puede ser sincrónica o asincrónica, con reportes de hasta 10-15 años sin evidencia de malignidad, por lo cual deben realizarse paraclínicos e imágenes de extensión y seguimiento periódico a largo plazo. En el grupo de la enfermedad de Paget extramamaria, la localización perianal tiene la mayor incidencia de malignidad subyacente relacionada, con tasas que varían del 33\% al 86\%, con mayor frecuencia de cánceres rectales y ováricos ${ }^{\left({ }^{15}\right)}$.

En este caso, la dificultad diagnóstica fue mayor debido a que la edad de la paciente está por fuera del rango de presentación típica de la patología.

El manejo de esta entidad se realiza mediante escisión local o cirugía radical, aunque no existe un consenso claro sobre este ${ }^{(16)}$. Otras medidas no quirúrgicas son la aplicación tópica de 5-fluouracilo o imiquimod al 5\% en crema, 3 veces a la semana, durante 16 semanas, o el tratamiento combinado de paclitaxel y trastuzumab. La radioterapia se indica en pacientes con enfermedad recurrente o residual ${ }^{(14)}$.

En este caso se optó por la cirugía micrográfica de Mohs, teniendo en cuenta la edad de la paciente, las menores tasas de recurrencia y la intención de ofrecer desde el inicio un tratamiento curativo. Se escogió el procesamiento por parafina, ya que cuando se realiza congelación se puede presentar vacuolización de las células epidérmicas y generar confusión con las células de la enfermedad de Paget; además, el bloque de parafina permite realizar inmunohistoquímica en los márgenes de resección. Es sabido que la recurrencia en la enfermedad de Paget perianal es del 30\% al 60\% y que con la cirugía de Mohs se ha logrado disminuir a un $25 \%{ }^{(17)}$.

\section{CONCLUSIÓN}

Se presenta el caso de una paciente joven con enfermedad de Paget extramamaria perianal primaria. Tanto la edad de presentación como la ubicación anatómica son poco frecuentes, por lo que inicialmente no se sospechó la condición; sin embargo, la realización de una biopsia en la primera valoración por dermatología permitió realizar el diagnóstico. Continúa siendo un gran reto para los dermatólogos el tratamiento y seguimiento de estos pacientes. 


\section{Puntos clave}

- La enfermedad de Paget es un adenocarcinoma intraepitelial de localización más frecuente a nivel mamario y más raramente en otras zonas anatómicas con presencia de glándulas apocrinas.

- La enfermedad de Paget perianal representa el 20\% de la enfermedad de Paget extramamaria, el 6,5\% de todas las enfermedades de Paget y solo el $1 \%$ de las neoplasias perianales.

- La relación con cáncer de mama subyacente es la regla en la enfermedad de Paget mamaria y en la forma extramamaria la asociación con neoplasia va del 4-86\%, siendo la localizada a nivel perianal la que cuenta con tasas más altas.

- El estudio y seguimiento de los pacientes debe ser a largo plazo ya que las neoplasias asociadas pueden presentarse antes, durante o después de la enfermedad de Paget, con reportes de hasta 15 años.

\section{REFERENCIAS}

1. Paget J. On disease of the mammary areola preceding cancer of the mammary gland. Am Cancer Soc J. 1974;21(5):303-4. doi.wiley.com/10.3322/canjclin.21.5.303.

2. Lopes Filho LL, Lopes IMRS, Lopes LRS, Enokihara MMSS, Michalany AO, Matsunaga N. Mammary and extramammary Paget's disease. An Bras Dermatol. 2015;90(2):219-31. doi: 10.159o/abd1806-4841.20153189.

3. Asel M, LeBoeuf N. Extramammary Paget's Disease. Hematol Oncol Clin North Am. 2018;8621(1):144-6. doi.org/10.1016/j.hoc.2018.09.003.

4. Gaurav A, Gupta V, Koul R, Dabas S, Sareen R, Geeta K, Arora V, Parikh PM, Aggarwal S. Practical consensus recommendatons for Paget's disease in breast cancer. South Asian J Cancer. 2018;7(2):83-6. doi: 10.4103/sajc.sajc_107_18.

5. Schmitt AR, Long BJ, Weaver AL, McGree ME, Bakkum-Gamez JN, Brewer JD, Cliby WA. Evidence-Based Screening Recommendations for Occult Cancers in the Setting of Newly Diagnosed Extramammary Paget Disease. Mayo Clin Proc. 2018;93(7):877-83. doi: 10.1016/j.mayocp.2018.02.024.
6. Merritt BG, Degesys CA, Brodland DG. ExtramammaryPagetDisease. DermatolClin.2019;37(3):261-7. doi.org/10.1016/j.det.2019.02.002.

7. Morris CR, Hurst EA. Extramammary Paget Disease: A Review of the Literature-Part I: History, Epidemiology, Pathogenesis, Presentation, Histopathology, and Diagnostic Work-up. Dermatol Surg. 2020;46(2):151-8. doi: 10.1097/DSS.0000000000002064.

8. Crocker, H. Radcliffe. Paget's disease, affecting the scrotum and penis [Internet]. R Coll Surg Engl. 1889. [citado 4 de mayo de 2020]; Disponible en: https://archive.org/details/b22280236/page/n3/ mode/2up.

9. Inglis K. Paget's Disease of the Nipple: With Special Reference to the Changes in the Ducts ${ }^{\star}$. Am J Pathol. 1946;22(1):1-33. PMCID: PMC1934155.

10. Muir R. The pathogenesis of paget's disease of the nipple and associated lesions. $\mathrm{Br} \quad \mathrm{J}$ Surg. 1935;22(88):728-37. doi.org/10.1002/bjs.1800228811.

11. Yim JH, Wick MR, Philpott GW, Norton JA, Doherty GM. Underlying pathology in mammary Paget's disease. Ann Surg Oncol. 1997;4(4):287-92. doi: 10.1007/BFo2303576.

12. Mengjun B, Zheng-Qiang W, Tasleem MM. Extramammary Paget's disease of the perianal re- 
gion: a review of the literature emphasizing management. Dermatol Surg. 2013;39(1):69-75. doi: 10.1111/dsu.12019.

13. Rajendran S, Koh CE, Solomon MJ. Extramammary Paget's disease of the perianal region: a 20-year experience. ANZ J Surg. 2017;87(3):132-7. doi.org/10.1111/ans.12814.

14. Devaji Rao S, Govindarajan M. Extramammary Paget's Disease of the Perianal Region. Indian J Surg. 2017;79(4):360-2. doi: 10.1007/s12262-017-1590-6.

15. Helwig EB, Graham JH. Anogenital (extramammary) Paget's disease. A clinicopathological study. Cancer. 1963;16:387-403. doi: 10.1002/1097-0142(196303)16:3<387::aid-cncr2820160314 >3.0.co;2-0.

16. Isik O, Aytac E, Brainard J, Valente MA, Abbas MA, Gorgun E. Perianal Paget's disease: three decades experience of a single institution. Int J Colorectal Dis. 2016;31(1):29-34. doi.org/10.1007/s00384-015-2342-3.

17. Hendi A, Brodland DG, Zitelli JA. Extramammary Paget's disease: Surgical treatment with Mohs micrographic surgery. J Am Acad Dermatol. 2004;51(5):767-73. doi: 10.1016/j.jaad.2004.07.004. 